\title{
The Effect of Prehistory on Elongational Viscosity and on Crystallization of Running Filament in Melt Spinning
}

\author{
Kiyohito KoYAMA and Osamu IsHIZUKA \\ Faculty of Engineering, Yamagata University, \\ 4-3-16 Jyonan, Yonezawa, 992, Japan.
}

(Received April 7, 1980)

\begin{abstract}
Three polypropylene samples (sample A, B, and B10) having different prehistory were prepared: sample $A$ was prepared by quenching the melt screw extrudated, and samples $B$ and B10 were prepared by isothermal crystallization from $1 \%$ solution and from $10 \%$ solution in xylene, respectively. The effects of prehistory on elongational viscosity and on crystallization were studied by a on-line measuring method in isothermal melt spinning and in melt spinning. The elongational viscosities were in the order sample $\mathrm{A}>$ sample $\mathrm{B} 10>$ sample $\mathrm{B}$. The difference in the results of online measurement between samples $A$ and $B$ is very evident in 'the second region' along the spinning way which is the region from $10 \mathrm{~cm}$ to $35 \mathrm{~cm}$ below the spinneret. In the last half of the second region, the elongational viscosity for sample B takes on a larger value than that of sample A. The crystallization for sample B begins at a point nearer to the spinneret or at higher temperature than that for sample A in this region, but the progress of crystallinity for sample A was more rapid than that for sample B. These results were compared with the data of quiescent crystallization, and are discussed in this paper in terms of the differences in melt structure and in crystal structure of polymers with different prehistories.

KEY WORDS Polypropylene / Prehistory / Elongational Viscosity / Crystallization / Isothermal / Melt Spinning / Melt Structure / Entanglement /
\end{abstract}

In a preceding paper, ${ }^{1}$ the effects of prehistory were studied by measuring melt rheological properties and the physical properties of the spun filaments of two polypropylene samples of different prehistory. The first sample (sample A) was prepared by quenching extrudated melt screw. The second sample (sample B) was prepared by solution crystallizing from a $1 \%$ xylene solution. The results of shear viscosity, die swell and spinnability of the melts showed differences according to prehistory: the melt properties were affected by the difference in entanglement between sample A and B. The measurements of thermal shrinkage and tensile testing showed that spun filament $\mathrm{A}$ had a larger fraction of tie chain and that spun filament $B$ was rich in the fold-type crystal fraction.

In this paper, the effect of prehistory is examined by on-line measurements of the running filaments having different prehistory. The elongational viscosity of a melt having a different prehistory was measured by isothermal melt spinning and by melt spinning. Crystallization behavior was studied for both samples A and B. The relationships between crystallization and melt structure or crysral structure is discussed.

\section{EXPERIMENTAL}

\section{Materials}

The samples investigated in this paper were similar to those prepared in our earlier paper ${ }^{1}$ and are summarized in Table I. The prehistory of the shear deformation was applied to each sample by a screw extruder; the extruded melt was then quenched in ice-water at the exit of the extruder (sample A). One half of an extruded sample was freed from the prehistory of shear deformation by dissolving it in xylene at $135^{\circ} \mathrm{C}$ and was then isothermally crystallized at $80^{\circ} \mathrm{C}$ from a $1 \%$ solution (sample B) and from a $10 \%$ solution (sample B10). No difference in the molecular weight among the three samples could be found and the molecular weight of 
Table I. Sample preparation

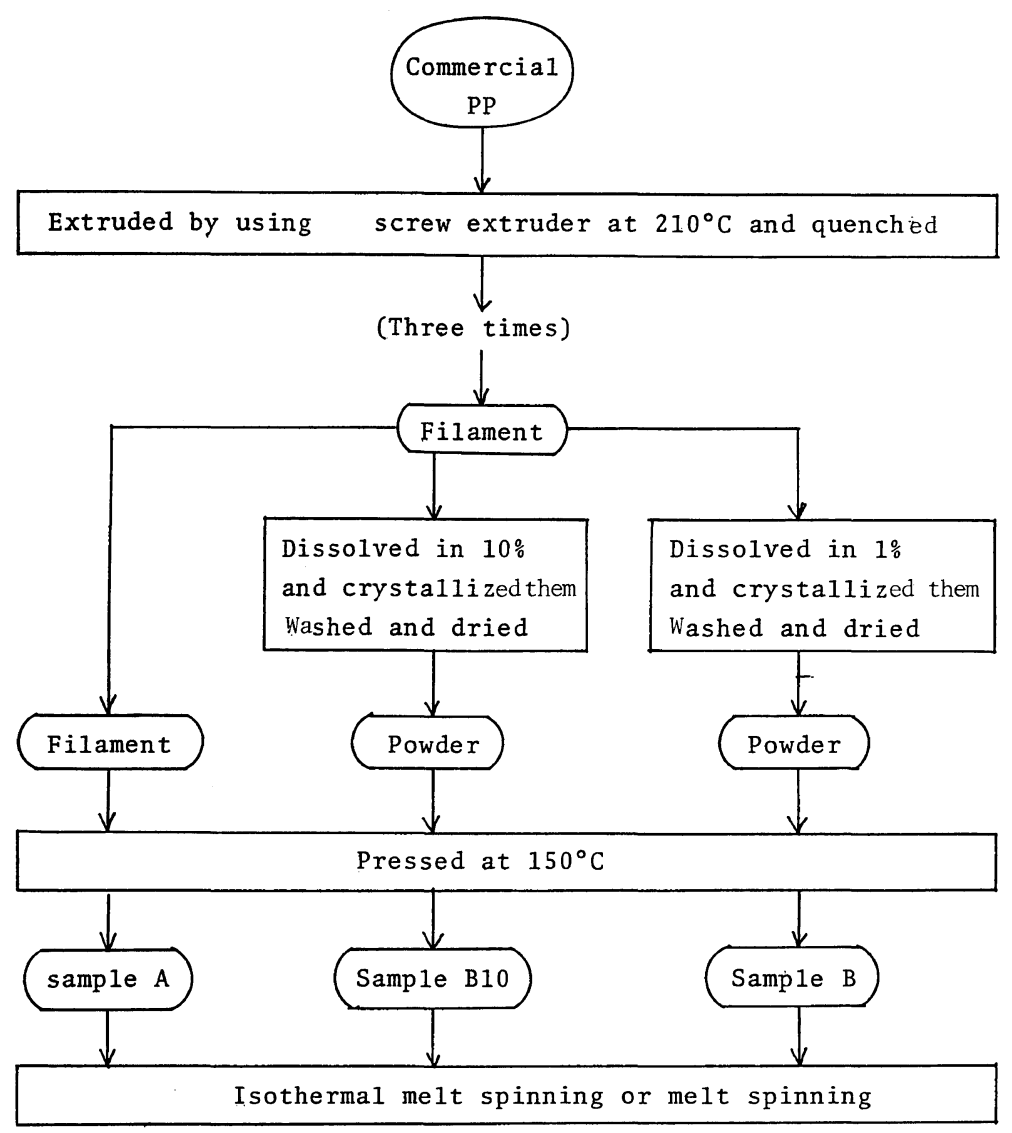

each was $1.5 \times 10^{5}$.

\section{Melt Spinning}

The melt-spinning apparatus and operation were previously described in some detail. ${ }^{2,3}$ The melt spinning temperature was $250^{\circ} \mathrm{C}$. The molten polymer was made to flow through a capillary by a piston motion of a constant speed. The capillary of the spinneret had a diameter of $2 \mathrm{~mm}$ and $\mathrm{L} / \mathrm{D}$ ratio of 20 and the extrusion rate was $0.0099 \mathrm{~cm}^{3} \mathrm{~s}^{-1}$. The take-up velocity was $67 \mathrm{~cm} \mathrm{~s}^{-1}$.

The isothermal melt-spinning apparatus and operation were a modification of that reported in greater detail in an earlier work. ${ }^{4}$ The extruded melt was passed through an electrically heated chamber maintained at a temperature of $180^{\circ} \mathrm{C}$, the same as that of the melt in the spinneret and then through ordinary room air wherein it was finally would onto a variable speed take-up device. The capillary diameter of the spinneret was $3.3 \mathrm{~mm}$ and $\mathrm{L} / \mathrm{D}$ ratio of 11 and the extrusion rate was $0.0032 \mathrm{~cm}^{3} \mathrm{~s}^{-1}$. The take-up velocity was $6.67 \mathrm{~cm} \mathrm{~s}^{-1}$. The isothermal zone length in this study was $13 \mathrm{~cm}$.

\section{On-Line Measurement}

The running filament was photographed through the microscope at a magnification from $20 \times$ to $100 \times$. The negative was magnified by an enlarger; the diameter $d$ of the running filament was then measured.

Filament tension measurements were carried out with a recording tensometer placed just above the take-up device. The rheological elongational force was calculated by the take-up force, gravitational constituent, aerodynamical constituent, and the inertial force in the force balance equation. ${ }^{5}$

The temperature of the running filament was measured by an IR Microscope (Model RM-2B in 
Barns Co. Ltd.), which is used for contactless temperature measurements. The temperature was measured by the comparing IR radiation emitted by the running filament with that emitted by the thermostatic black body. Objective lens magnifications were $15 \times$ and $52 \times$.

Wide angle X-ray diffraction patterns on the running filaments were obtained along the spinning direction as far as $100 \mathrm{~cm}$ from the spinneret. In this measurement, the $\mathrm{X}$-ray was conducted on the moving filament during processing. $\mathrm{Cu}-\mathrm{K} \alpha$ radiation through an $\mathrm{Ni}$ filter was used. A pinhole collimated X-ray beam was used, and the diffracted $\mathrm{X}$-ray was detected by a scintillation counter and pulse-height discrimination. The separation of amorphous scattering from the crystalline peaks was made by the method suggested by Weidinger and Hermans. ${ }^{6}$

\section{Static Crystallization}

The growth rate of spherulites was calculated by measuring the size of the spherulites as a function of time during the isothermal crystallization process. Optical microscopy, using crossed polarizers and two thermostatted hot-stages, were utilized for this study. Development of the spherulites was followed by photographing them at appropriate intervals. Isothermal crystallization rates of the polymers were determined from the dilatometetrically measured changes in volume with time.

\section{RESULTS AND DISCUSSION}

\section{Isothermal Melt Spinning}

The characteristics of molten polymers can be studied by shear viscosity, die swell, and elongational viscosity, in general. The shear viscosity and the die swell for samples A and B were examined in previous study. ${ }^{1}$

Sample A shows a viscosity and die swell ratio greater than that of sample B. In this paper, the elongational viscosities of these samples are studied by the isothermal melt-spinning method.

A typical diameter profile in isothermal melt spinning is shown in Figure 1. The solid line indicates the data within the isothermal zone. The diameter profiles for samples $\mathrm{A}, \mathrm{B}$, and $\mathrm{B} 10$ are similar. The extruded molten polymer shows a maximum diameter which then becomes attenuated, and this attenuation of filament diameter, occurs throughout the outer region of the isothermal zone. However, the swell ratio of sample B is slightly smaller than that of sample $A$ and this is in accordance with previous results. ${ }^{1}$ The swell ratio of sample B10 is intermediate between samples B and A.

The filament attenuation process for sample $B$ is more rapid than that for sample B10, and that for sample B10 is more rapid than that for sample A in the isothermal zone. Thus, the elongational strain

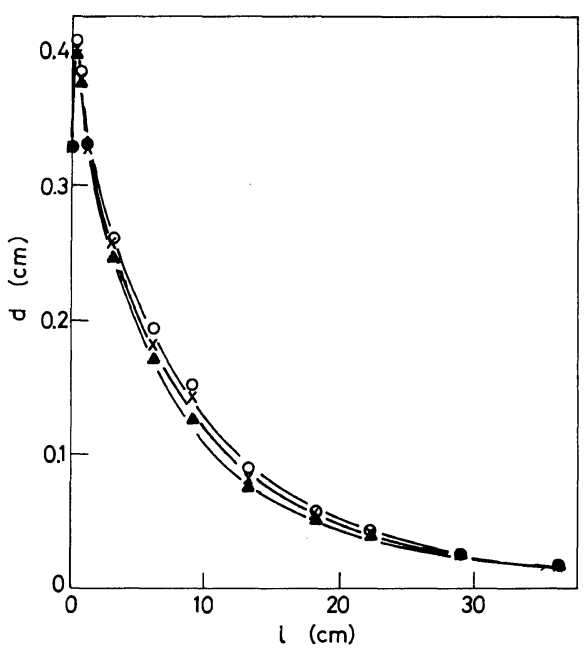

Figure 1. Variation in filament diameter along the spinning direction in isothermal melt spinning for sample A (.O), sample B10 ( $\times$ ), and sample B (A).

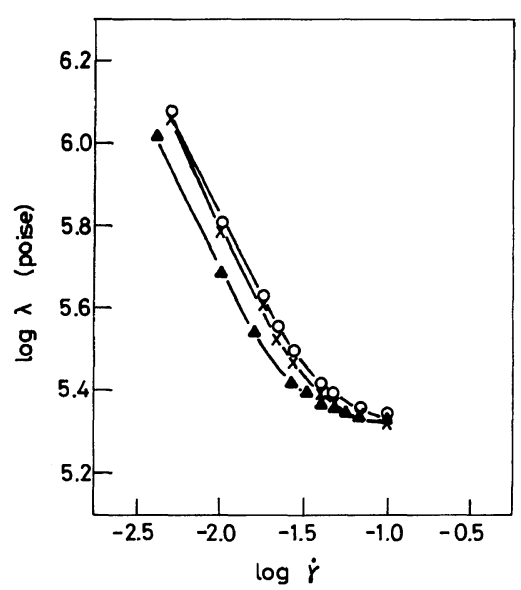

Figure 2. Relationships between elongational viscosity and elongational strain rate in isothermal melt spinning for sample A (O), sample B10 (x), and sample B (A). 
rate for sample B shows a value in the isothermal zone larger than that for samples B10 and A. When the length of the isothermal zone was extended, the molten filaments broke. Thus, we could not find the experimental conditions such that samples with the fully developed elongational flows could be obtained. Such experimental conditions, however, could be found for higher-molecular-weight polypropylene. $^{4}$

Elongational viscosity was calculated by the elongational strain rate, rheological elongational force, and filament diameter. Figure 2 illustrates the variation in elongational viscosity with the elongational strain rate for samples $\mathrm{A}, \mathrm{B}$, and $\mathrm{B} 10$. The elongational viscosity decreased with strain rate, and in the case of sample B the viscosity had a constant value at a higher strain rate. At a lower strain rate, the elongational viscosities were in the order sample $\mathrm{A}>$ sample $\mathrm{B} 10>$ sample $\mathrm{B}$. The relationship between samples $A$ and $B$ is similar to that in shear viscosity $v s$. shear strain rate. ${ }^{1}$ This similarity is associated with deformation, which is a certain similar flow to shear, within the filament in the neighborhood of the spinneret. ${ }^{4}$

The smaller values for the elongational viscosity of sample B relative to sample A indicate that a molecule in the melt of sample B is less interactive with other molecules, as a result of fewer entanglements.

The elongational viscosity for sample B10 is intermediate between those for samples $\mathbf{B}$ and $\mathbf{A}$. Sample B10 was prepared by dissolution in a 10\% solution of xylene and by being crystallized from this solutions; sample B was prepared from a $1 \%$ solution of xylene.

The difference in solution concentration $(1 \%$ and $10 \%$ ) affects the elongational viscosity in the melt. Moreover, the viscosity of sample B10 prepared from a $10 \%$ solution of xylene shows a larger viscosity value than that of the $1 \%$ solution, being rather close to that of sample A which has a prehistory of shear deformation. It is considered that the molecules in the $1 \%$ solution are well separated and can extract themselves from solution so as to fit into the crystal, but in the $10 \%$ solution such separation and extraction is incomplete. These differences in solution still remain ever after melting. The melt of sample B10 is richer in molecular entanglement and yields a higher elongational viscosity than sample $B$.
In the range of a higher strain rate or larger strain of the isothermal melt spinning, the elongational viscosity for sample $B$ remains constant and taken on the same value as sample A and B10. This behavior in sample $\mathrm{B}$ belongs to the second region of isothermal melt spinning in previous investigation. ${ }^{4}$ Because sample $\mathrm{B}$ undergoes a larger deformation in isothermal zone and since the degree of the development of elongational flow is greater in sample B than that of other samples, the second region in isothermal melt spinning ${ }^{4}$ may appear only in sample B.

\section{Elongational Behavior in Melt Spinning}

The filament diameter and the elongational strain rate versus distance from the spinneret in melt spinning are shown for samples A and B in Figure 3. Observation reveals that filament attenuation proceeds through three stages. The first stage is in the vicinity of the spinneret $(\sim 10 \mathrm{~cm})$. The second stage is the region from $10 \mathrm{~cm}$, to $35 \mathrm{~cm}$, and the third stage is that region from $35 \mathrm{~cm}$ up to the take-up position.

In the first and the third regions, no significant difference in filament diameters for samples A and B can be detected, nor is it measurable. In the second region, the filament diameter profiles and that of the elongational strain rate for sample A differ significantly from those for sample B. The attenuation of

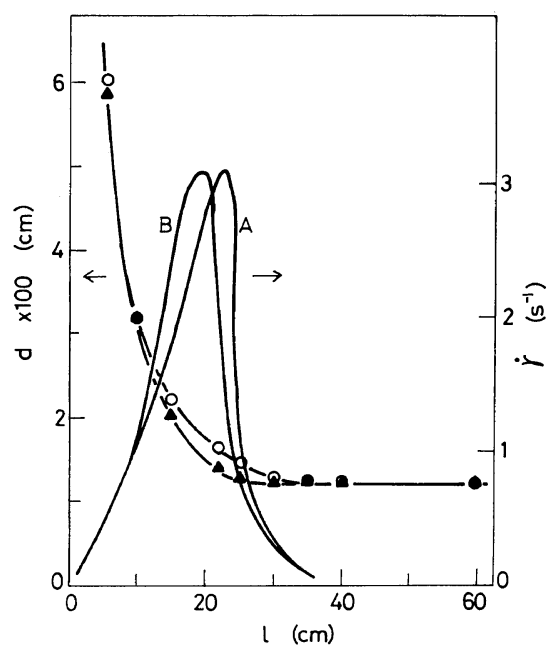

Figure 3. Variation in filament diameter and elongational strain rate along the spinning direction in melt spinning for sample A $(\bigcirc)$ and sample B $(\boldsymbol{A})$. 


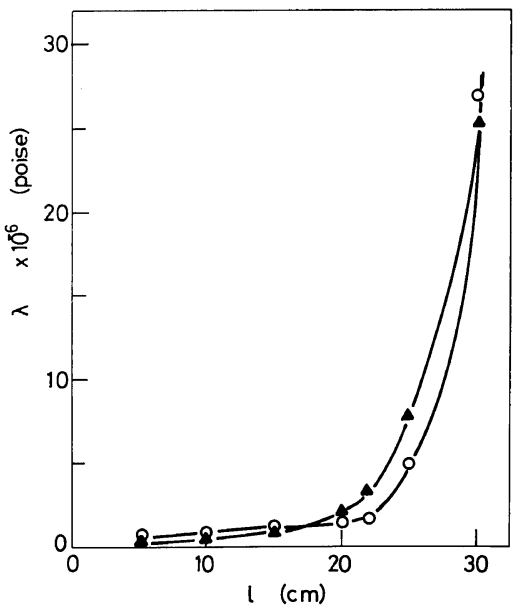

Figure 4. Variation in elongational viscosity along the spinning direction in melt spinning for sample $A(O)$ and sample B ( $\mathbf{\Delta})$.

sample B is rapid at a point nearer to the spinneret in the second region, compared with that of sample A. The features of the diameter profile in melt spinning are the same as those shown isothermal melt spinning. The elongational strain rate of sample B shows a value larger than that of sample A at a point nearer the spinneret in the second region. The maximum position of elongational strain rate for sample B is situated at a shorter distance from the spinneret than that of sample A. The positions indicating the very small values of elongational strain rate are almost same for both samples.

Figure 4 shows the elongational viscosity $v s$. the distance from the spinneret in melt spinning for sample $\mathrm{A}$ and $\mathrm{B}$. The elongational viscosity for sample B is smaller than for sample A in the first region. The larger elongational strain rate of sample $\mathbf{B}$ in this region (Figure 3 ) is brought about by this small elongational viscosity. This behavior of samples A and B in melt spinning is the same as that in isothermal melt spinning, suggesting that molten sample B possesses a smaller content of molecular entanglement than sample A. On the contrary, the elongational viscosity in the far region situated $20 \mathrm{~cm}$ from the second region of sample B is higher than that of sample A. This higher elongational viscosity causes the small elongational-strain rate of sample $B$ in this region (Figure 3). In the third region the elongational viscosity of sample $B$ is almost same as that of sample A.

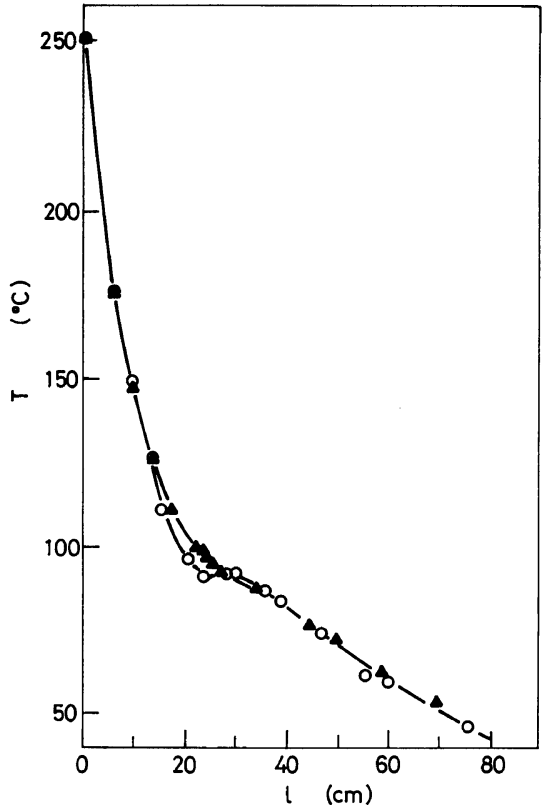

Figure 5. Temperature distribution of running filament in melt spinning for sample A $(O)$ and sample B (A).

\section{Temperature Distribution of the Running Filament}

Filament temperature $v s$. the distance from the spinneret is shown for samples A and B in Figure 5. The filament cools rapidly in the first and the third regions of the preceding section and in these regions, no difference in temperature distribution between samples A and B could be detected. In the second region, the cooling curves for samples $\mathrm{A}$ and $\mathrm{B}$ manifest the different features. The running filament for sample B cools slowly but for sample A, this cooling is initially rapid and then reaches a plateau region. This pronounced difference between sample $A$ and $B$ in the second region also occurs in the case of the filament diameter and elongational strain rate. The filament diameter for sample $B$ was smaller than that of sample A in the second region (Figure 3). This smaller filament diameter results in rapid cooling. ${ }^{7}$ Therefore, it is expected that sample B has a lower filament temperature than sample A. In actuality, the measured temperature for smple B is larger than that for sample A. It is thus necessary to take into account factor other than the cooling rate.

Figure 6 shows a plots of elongational viscosity as a function of the reciprocal of temperature. It can be 


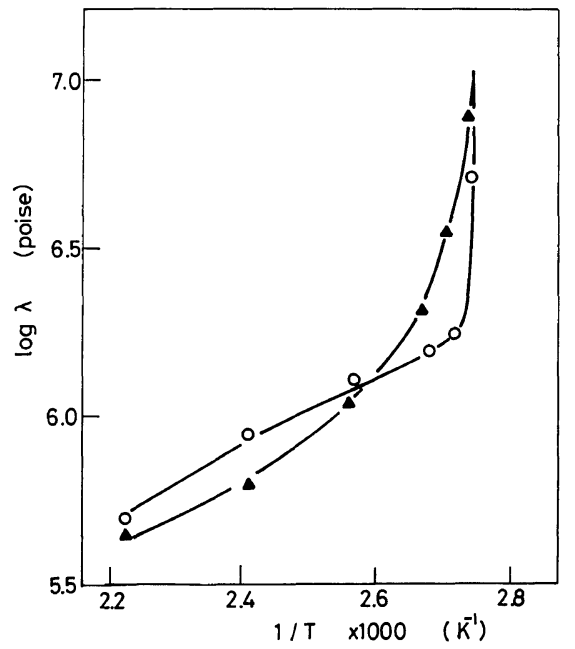

Figure 6. Relationships between elongational viscosity and the reciprocal of temperature for sample $A(O)$ and sample $B(\boldsymbol{\Delta})$ in melt spinning.

seen that at higher temperatures the elongational viscosity for sample $A$ is larger than that for sample B; the presence of entanglements may have an effect. At lower temperatures (the second region), the elongational viscosity for sample $\mathrm{A}$ is runs counter to expection from prehistory and is smaller than that for sample B at the same temperatures. These results suggest that a significant difference in flow structure between sample A and B exists in the second region.

\section{Crystallization Behavior of Running Filament}

The on-line X-ray diffraction experiments for running filaments were carried out so as to study structural change or crystallization behavior. In the first region of the spinning way, $\mathrm{X}$-ray diffraction curves show halos for both samples A and B, meaning that the running filaments are still molten and have not yet crystallized in this region.

Typical X-ray diffraction curves of samples A and $B$ in the second region are shown in Figure 7. At $20 \mathrm{~cm}$ from the spinneret, the X-ray diffraction curve for sample $\mathrm{A}$ is a halo and that for sample $\mathrm{B}$ shows $\alpha$-phase crystalline peaks. It is clear that the crystallization for sample B starts earlier than that for sample A. At $30 \mathrm{~cm}$ from the spinneret, the Xray diffraction curves show well developed crystalline peaks for both samples and the degree of crystallinity at $30 \mathrm{~cm}$ is $2 / 3$ of that for the take-up

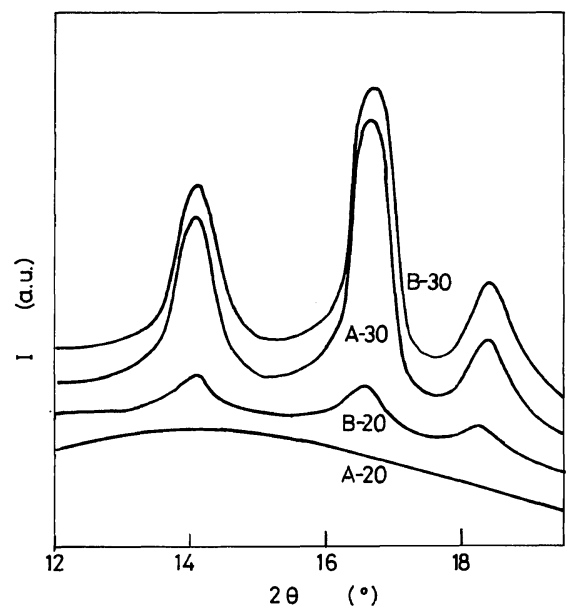

Figure 7. X-ray diffraction curves of running filament at distances of $20 \mathrm{~cm}$ and $30 \mathrm{~cm}$ from the spinneret for sample $\mathrm{A}$ and $\mathrm{B}$.

filament. No difference in $\mathrm{X}$-ray diffraction curves between samples $A$ and $B$ is recognized at $30 \mathrm{~cm}$. The residence time from $20 \mathrm{~cm}$ to $30 \mathrm{~cm}$ is about $0.15 \mathrm{~s}$. The first half of crystallization proceeds very fast for both samples but the crysrallization rate for sample A is faster than that for sample B. Namely, although the start of crystallization for sample $A$ is retarded, the progress of crystallization for sample A is more rapid than that of sample B in the first half of the crystallization process. This fast progress of crystallization for sample A makes way for the following interpretation, that more entanglements or tie points of sample A under elongational stress increase the orientation of the chain and enhance the difference in entropy between the melt and the crystal. This reduction of the entropy results in a rapid acceleration of the crystallization transformation. However, the earlier starting of crystallization of sample B cannot be intuitively explained on the basis of the effect of elongational deformation on the nucleation rate or crystal-growth rate.

In the third region, the running filaments are crystallized slowly and the degree of crystallinity reaches a constant value $(=60 \%)$ at $70 \mathrm{~cm}$ from the spinneret. The latter half of the crystallization process in running filament is the same in both samples.

$\mathrm{X}$-ray diffraction results for sample $\mathrm{A}$ are almost the same as those for the commercial pellets in a 


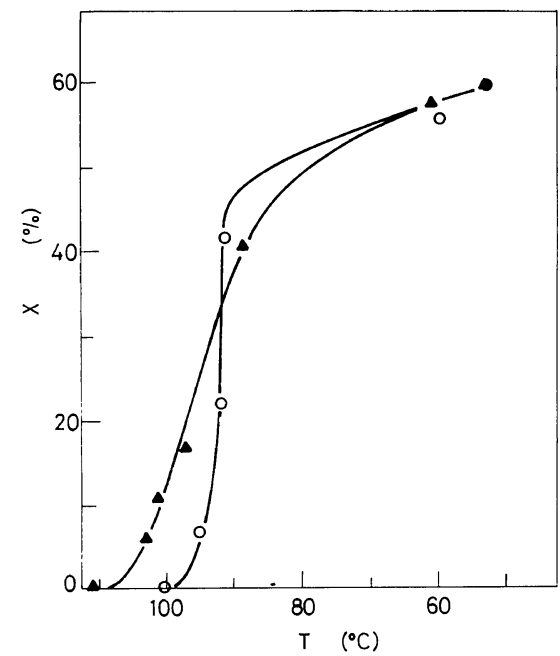

Figure 8. Plots of crystallinity of running filament $v s$. temperature for sample $\mathrm{A}(\mathrm{O})$ and sample $\mathrm{B}(\boldsymbol{\Delta})$.

previous study. ${ }^{3}$ The diameter profile and the temperature distribution for sample A differ from that for sample $\mathrm{B}$ in the second region, and in the third region, no difference in the diameter profile and in the temperature distribution for both samples can be recognized. Therefore, results for sample A and B obtained by different measuring methods are compatible with each other.

Figure 8 shows the plots of the degree of crystallinity $v s$. temperature of running filament. Sample B slowly crystallizes over a wider range of temperature and sample A crystallizes to a great extent at a constant temperature. The explanation for this is that following the onset of crystallization the effect of elongational deformation on the molecular orientation of sample $\mathrm{A}$ is larger than that for sample B. This molecular orientation accelerates the crystallization of sample A. The rapid progress of crystallization for sample A results in large release of crystallization heat. The filament temperature for sample A may thus be kept constant because of the balance between heat released by crystallization and that lost by cooling. Actually, the temperature of sample $\mathrm{A}$ in the second region shows a constant value in Figure 5.

A further feature shown in Figure 8 is that sample B starts to crystallize above $100^{\circ} \mathrm{C}$ and sample A starts to crystallize below this temperature. This is difficult to explain merely by the effect of orientation

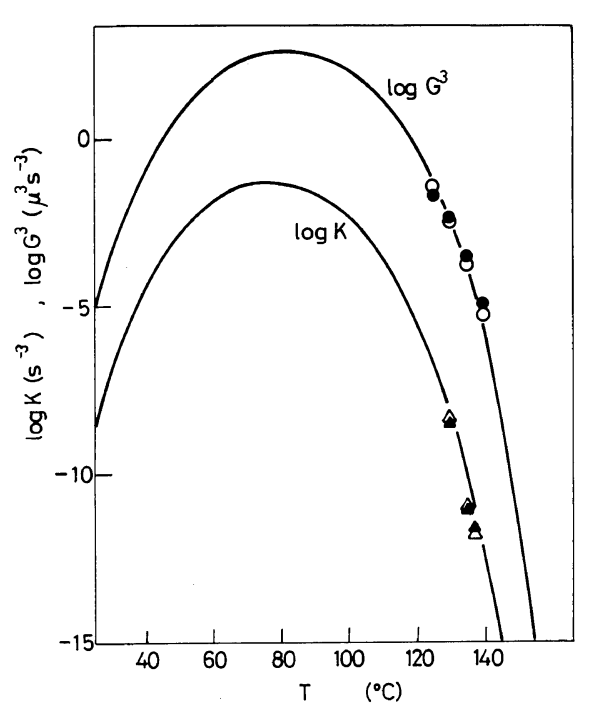

Figure 9. Plots of crystallization rate constant $(K)$ and growth rate constant $(G)$ vs. crystallization temperature for sample $\mathrm{A}(\triangle, O)$ and sample $\mathrm{B}(\boldsymbol{\Delta}, \boldsymbol{O})$. Solid line is theoretical curves.

on crystallization, since sample B is free from deformation prehistory and possesses fewer entanglements or tie points than sample A.

Sample B crystallizes at a higher temperature and becomes a mixture of crystallite and supercooled melt at a higher temperature than sample A. This mixture of sample B at a higher temperature yields high viscosity in the latter half of the second region. This explains the rapid increase in viscosity for sample B at higher temperatures (Figure 6) or a point nearer to the spinneret (Figure 4). This higher viscosity causes the attenuation to become more rapid near to the spinneret (Figure 3). The slow start of crystallization of sample A prevent a rapid increase in viscosity (Figures 4 and 6).

\section{Crystallization Rate Constant}

In order to study the development of crystallinity in a quiescent polymer melt for samples $\mathrm{A}$ and $\mathrm{B}$, two types of experiments were carried out. One was concerned with assessing the isothermal rate $(K)$ at which the total amount of crystallinity develops from a supercooled liquid and is measured by dilatometry. The other was the direct determination of the isothermal rate of spherulite growth $(G)$ by means of light microscopy. Temperature dependence of the total crystallization-rate constant and 
the growth-rate constant are shown in Figure 9, along with theoretical curves. ${ }^{2}$

To discuss the influence of prehistory, namely melt structure, and that of crystal structure on the crystallization process, it is convenient to separate the crystallization-rate constant and the growth-rate constant expression into three parts:

$$
\begin{aligned}
& \log K=\log K_{0}-\frac{\Delta E}{k T}-\frac{\Delta F}{k T} \\
& \log G=\log G_{0}-\frac{\Delta E^{\prime}}{k T}-\frac{\Delta F^{\prime}}{k T}
\end{aligned}
$$

The last two terms in both equations represent the effect of free energy for activation on the transport of crystallizing units across the phase boundary and formation of the critical size nucleus. The first term is independent of the melt and crystal structures.

The third term is a dominant factor in either the higher temperature or lower supercooling range, for which experimental data in the quiescent melt are shown in Figure 9. The free energy for forming critical nucleus is divided into the heat of fusion, the term concerning entropy of fusion and the interfacial free energy at a constant temperature. ${ }^{8}$ The difference in the former two factors between samples A and $\mathrm{B}$ is little because the melting temperatures is the same for both samples. While previous results for melt spun filament showed that sample A tends to growth in the form of a fringed micellar crystal and sample $B$, in the form of a chain-folded type crystal. The difference between the two crystalgrowth types mainly appears in the interface free energy of the surface, especially in the end surface free energy $\left(\sigma_{\mathrm{e}}\right)$. It has been also considered that the end surface free energy of the fringed micellar nucleus (which prevails in sample $\mathrm{A}$ ) is larger than the corresponding quantity for folded nuclei (which will prevail in sample B). ${ }^{8,9}$ Thus, one may expect that the crystallization constant and growth-rate constant for sample A are smaller than those for sample B. However, these differences are hardly recognizable, except for the slightly larger value in the growth-rate constant of sample B. This is explained by the fact that at a much lower supercooling temperature corresponding to the right region of the maximum in curves in Figure 9, crystallization requires a long enough time to disentangle the long-chain molecules and to transform these molecules from supercooled melt to the crystal form. During the slow crystallization process, the prehistory of samples A and B in melt disappears and no difference in crystallization-rate constant is observed.

At a larger supercooling temperature corresponding to the left region of the maximum in curves in Figure 9, the second term in eq 1 becomes more important. The different melt structure affects the second term related to the transport of long-chain molecules from the melt to the crystal surface. The melt viscosity of sample B is smaller than that of sample A. In the crystallization process, the viscosity of sample B is considered to be small and the transport of crystallizing molecules occurs more readily in sample $B$ than in sample $A$. Thus, the crystallization-rate constant for sample $B$ is expected to be larger than that for sample A, but no experimental data in this temperature range could be obtained.

The crystallization of the running filament occurs within $90-110^{\circ} \mathrm{C}$ and is situated slightly at the right of the maximum in curves in Figure 9. Since the temperature corresponding to the maxima of the crystallization and the growth-rate constants shifted to a higher temperature in the case of crystallization from oriented melt, the crystallization of the running filament can almost be regarded to occur at the temperature at which the curves shifted. At this temperature, both the second and the third terms are important and the time required for crystallization is very short. The second term is closely associated with the melt viscosity or melt relaxation time. The melt of sample A has a higher viscosity (Figure 2) and its relaxation time is larger than that of sample B. ${ }^{1}$ The molecules of sample $\mathrm{A}$ are not thus considered to have sufficient mobility in the time scale of crystallization to proceed from the melt conformation to the chain-folded macroconformation. This results in the fringed micellar-type crystallization of sample A, and well explains the difference in physical properties of the spun filaments for samples A and B. ${ }^{1}$ Since sample B is considered to have few interpenetrated molecules, it tends to crystallize with the chain folds. The ease of molecular movement for the molten sample B hastens its crystallization. Furthermore, the endsurface free energy of the fold-type crystal is smaller than that of the fringed micellar crystal. The small end surface free energy of sample B accelerates the start of crystallization. This explains well the higher 
crystallization rate of sample $\mathbf{B}$ at higher temperatures.

Acknowledgement. The authors are indebted to Messrs. K. Ito and M. Sato for their assistance in some of the experiments. This work was supported by the Grant-in-Aid for Scientific Research from the Ministry of Education, Science and Culture in Japan.

\section{REFERENCES}

1. O. Ishizuka and K. Koyama, Sen'i Gakkaishi, 35 T453 (1979).
2. O. Ishizuka and K. Koyama, Polymer, 18, 913 (1977).

3. O. Ishizuka and K. Koyama, Sen'i Gakkaishi, 32, T43 (1976).

4. O. Ishizuka, K. Koyama and H. Nokubo, Polymer, 21, 691 (1980).

5. A. Ziabicki, Kolloid Z., 175, 14 (1961).

6. A. Weidinger and P. H. Hermans, Makromol. Chem., 50, 98 (1961).

7. A. Ziabicki, "Fundamental of Fiber Formation," John Wiley and Sons, London, 1976, Chapter 3.

8. J. D. Hoffman and J. I. Lauritzen, J. Res. NBS, 65A, 197 (1961).

9. P. H. Geil, "Polymer Single Crystal," Interscience, New York, N.Y., 1963, p 388. 